QUARTERLY OF APPLIED MATHEMATICS

VOLUME LXIX, NUMBER 4

DECEMBER 2011, PAGES 771-786

S 0033-569X(2011)01242-8

Article electronically published on July 12, 2011

\title{
THREE-PHASE ECCENTRIC ANNULUS SUBJECTED TO A POTENTIAL FIELD INDUCED BY ARBITRARY SINGULARITIES
}

\author{
BY \\ YU. V. OBNOSOV \\ Institute of Mathematics and Mechanics, Kazan State University, Prof. Nughin Str.,1/37, Kazan, \\ 420008, Russia
}

\begin{abstract}
An infinite planar, three-component heterogeneous medium with a pair of circles as interfaces between homogeneous zones forming an eccentric annulus is considered for refraction of a potential field on the two interfaces. The velocity field is generated by an arbitrary system of singularities of arbitrary order, in congruity with the MilneThomson case of a two-component medium and a single circular interface. An exact analytical solution of the corresponding $\mathbb{R}$-linear conjugation problem of two Laplacian fields in the eccentrical annulus structure is derived in the class of piecewise meromorphic functions with fixed principal part. Three general cases of loci of the singularities with respect to the interfaces are investigated. Flow nets (isobars and streamlines) are presented.
\end{abstract}

1. Introduction. Composite (heterogeneous) materials are common in nature (e.g. soils and rocks, Dagan [2]), in modern designed engineering constructions (e.g. tessellations of doped semi-conductors, Milton [15]) and in engineering control (modification) of natural environments where fascinating composite patterns are formed with anthropogenic interventions (De Zwart [4, Obnosov et al. 20]). The induced or existing fields of pressure, hydraulic head, electrical current, concentration, temperature, which are described by the Darcy, Ohm, Fick, Fourier laws, in these composites are amazingly complex, counterintuitive and puzzling in some characteristics (e.g. topology of streamlines). Moreover, if the engineer or designer can control the position of field-inducing singularities and/or conducting properties of the components of the composite, an unexpected optimality of integral characteristics (e.g. total flow rate or total current) has been revealed (Obnosov et al. [20]).

It is remarkable that the same mathematical model describes the different physical fields mentioned above. In what follows we shall use the language of Darcian flows in

Received May 3, 2010.

2010 Mathematics Subject Classification. Primary 30E25, 76T30.

Key words and phrases. Refraction, heterogeneous media, $\mathbb{R}$-linear conjugation problem, analytic functions.

E-mail address: Yurii.Obnosov@ksu.ru

(C)2011 Brown University Reverts to public domain 28 years from publication 
porous media (Strack 24]). Then, in order to solve the problem of fluid motion, it is required to determine a piecewise-holomorphic vector field $\mathbf{w}=\left(w_{x}, w_{y}, w_{z}\right)=\mathbf{w}_{p}$ such that

$$
\operatorname{div} \mathbf{w}_{p}=0, \quad \operatorname{curl}\left(\rho \mathbf{w}_{p}\right)=0
$$

in all the uniform components $S_{p}$ of a considered medium:

$$
\left(\mathbf{w}_{p}\right)_{n}=\left(\mathbf{w}_{q}\right)_{n}, \quad\left(\rho_{p} \mathbf{w}_{p}\right)_{\tau}=\left(\rho_{q} \mathbf{w}_{q}\right)_{\tau}
$$

along the boundary dividing unlike components $S_{p}$ and $S_{q}$. Here $\mathbf{w}_{n}$ and $(\rho \mathbf{w})_{\tau}$ are the normal and tangential components of the corresponding limit boundary values of vectors $\mathbf{w}$ and $\rho \mathbf{w}\left(\rho \equiv \rho_{p} \equiv\right.$ const is a constant parameter, characterizing the component $S_{p}$ property).

The general refraction problem (12), (2) is utmost difficult for analytical solution and admits such a solution for some specific structures only. So, various asymptotic, approximate, and numerical approaches were utilized (see a recent book by Milton [15] and the article by Drygaś, Mityushev [5] for comprehensive references).

The current paper deals with a plane heterogeneous structure with circular interfaces between zones of contrasting conductivity $k=1 / \rho$, which has attracted the attention of many investigators. Beginning from Rayleigh [23] and Maxwell [13, composites with circular inclusions were intensively studied. In what follows we reference only some works either containing a detailed bibliography or directly connected with this paper.

If a "background" plane has a finite number of nonconducting inclusions of arbitrary shapes, then the corresponding solution of the problem (11), (2) can be found as a conformal mapping of an infinite "matrix" with "holes" (no-flow contours) onto the $w$-plane with slots parallel to the real axis. The most impressive progress here was done by Crowdy [1. He has considered an important problem of streamline distribution for a uniform irrotational flow, caused by the given dipole at infinity, past a multi-cylinder configuration. Lee [11 has found a solution for a problem of a flow past two circular inclusions when a given undisturbed (i.e. with no inclusions) complex potential $f(z)$ has a finite number of point vortices and a dipole at infinity.

If inclusions are conducting (but differently from the matrix), then the solution of the problem (11), (2) is more complicated. The case of two conducting circular inclusions is the most intensively studied. Different particular cases of the two circular inclusions with a fixed dipole at infinity were studied in a series of papers by Emets and Obnosov, one of which [7] was duplicated in [25]. A general solution of the last problem, including all degenerate and limit cases, was derived in [17. Palaniappan [21] has investigated the perturbation of a given electric field by two intersecting conducting cylinders.

E. Honein et al. 9, 10, have considered the conjugation problem for two nonintersecting elastic circular inclusions perfectly bonded to an elastic matrix subjected to arbitrary loads (singularities) that produce an anti-plane deformation. We emphasize that in 9] the solution was derived only for the case when there are no singularities of $f(z)$ inside both circles. Though Honein et al. declared that the situation when $f(z)$ has singular points inside inclusions as well as the case when one inclusion is situated inside another could be easily considered, as far as we know, this has not been done. We believe that the lack of explicit solutions to the case not covered in Honein et al. can be explained in the 
following manner. In [9], a required solution was found using the method of images and the generalized Milne-Thomson circular theorem. But the last theorem till recently was rigorously proved only for the case when all singular points of $f(z)$ are located outside the inclusion (see [8, 22, p. 351], and later articles cited in [9]). The general case of the Milne-Thomson circular theorem with arbitrary distributed singularities of the given complex potential was completely cracked only recently in [16. In [12] we considered the case of a concentric annulus and arbitrary $f(z)$.

The present article is the natural generalization of the works [9, [16, 12] to the case of an eccentric annulus and arbitrary undisturbed complex potential. In contrast to [9] we consider the problem of a double circular inclusion (Fig. 1) when one inclusion is inside another. This case is especially important in well hydraulics (both in groundwater hydrology and reservoir engineering, Polubarinova-Kochina 22], De Zwart [3], Obnosov et al. 20]). The internal circle in Fig. 1 corresponds to the interface between a gravel pack in a drilled borehole and the formation. The external circle in Fig. 1 is the boundary up to which a drilling liquid (mud) infiltrated from the borehole during drilling. The zone between the two circles is often called the "formation damage". The domain exterior to the second circle is the background matrix (formation) which was not affected by drilling. The simplest type of singularity in Fig. 1 is a sink which corresponds to the screen of an abstraction well. This kind of sink is always inside the gravel pack. The source (normally in the matrix) paired with the sink is commonly an injection well (secondary oil recovery or pump-and-treat system in contaminant hydrology). The main practical issue for Darcian flows is to understand how the eccentricity, the position of singularities and the conductivities $k_{1}, k_{2}$ and $k_{3}$ of the three zones in Fig. 11influence the flow topology in the vicinity of the formation damage.

2. Formulation. A planar infinite matrix in Fig. 1 is considered as a complex plane $\mathbb{C}$ of a variable $z$ (the origin of coordinates coincides with the center of the external circumference). The function $f(z)$ is a given complex potential with a finite number of singularities. As we have discussed this "background" potential (an arbitrary function) characterizes the homogeneous case, from which in subsurface mechanics all flow calculations start by adding the potential perturbance caused by heterogeneities (PolubarinovaKochina [22]). In our case, the problem is to determine the disturbed complex potential $w(z)$ after insertion of a two-component asymmetric circular inclusion into $\mathbb{C}$ such that the medium becomes three-component. We stress that the "background" singularities of $f(z)$ are situated arbitrarily with respect to the inclusion in Fig. 1

In terms of a piecewise meromorphic function $v(z)=w^{\prime}(z)=v_{x}(x, y)-\mathrm{i} v_{y}(x, y)$, complex conjugated with the complex velocity function $\mathbf{v}(z)=v_{x}(x, y)+\mathrm{i} v_{y}(x, y)$, this problem is equivalent to an $\mathbb{R}$-linear conjugation problem [6, p.53]. Namely, let $S_{1}=\{z$ : $\left.|z|>r_{1}\right\}, S_{2}=\left\{z:|z|<r_{1},|z-h|>r_{2}\right\}$ and $S_{3}=\left\{z:|z-h|<r_{2}\right\}$ be the infinite matrix, eccentric circular annulus and circle inside the annulus respectively (Fig. 1). The function $v(z)=v_{k}(z), z \in S_{k}$ is meromorphic in $S_{k}$ and continuous in $\bar{S}_{k}$ everywhere, except at singular points of its principal part, which coincide with the corresponding singular summands of $f^{\prime}(z)(k=1,2,3)$. Then the limit values of $v_{k}(z)$ at the boundary 
$\partial S_{2}$ are conjugated with the conditions

$$
\begin{aligned}
& v_{2}(t)=A_{21} v_{1}(t)+B_{21} r_{1}^{2} t^{-2} \overline{v_{1}(t)}, \quad t \in \mathcal{L}=\left\{t:|t|=r_{1}\right\}, \\
& v_{2}(t)=A_{23} v_{3}(t)+B_{23} r_{2}^{2}(t-h)^{-2} \overline{v_{3}(t)}, \quad t \in \ell=\left\{t:|t-h|=r_{2}\right\},
\end{aligned}
$$

where

$$
A_{p q}=\frac{\rho_{p}+\rho_{q}}{2 \rho_{p}}, \quad B_{p q}=\frac{\rho_{p}-\rho_{q}}{2 \rho_{q}}, \quad \Delta_{p q}=B_{p q} / A_{p q}=\frac{\rho_{p}-\rho_{q}}{\rho_{p}+\rho_{q}} .
$$

Here $\rho_{p}\left(k_{p}=1 / \rho_{p}\right)$ is the resistivity (conductivity) of a medium $S_{p}$.

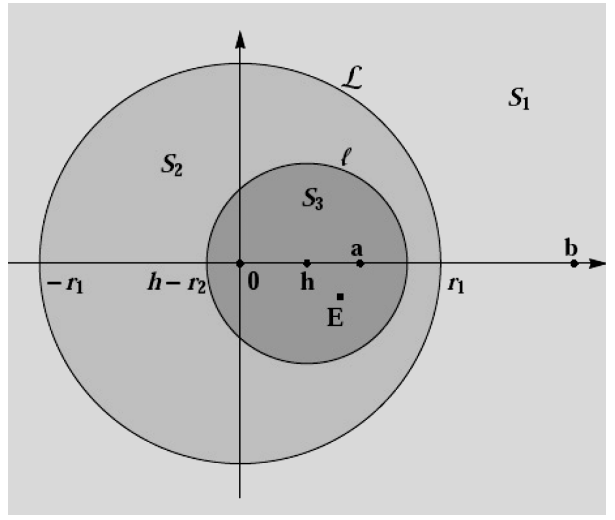

FIG. 1. Asymmetric annulus

We start with a solution of the problem (3) under the assumption that $f(z)$ has a single singularity at a point $E \in S_{3}$.

3. Solution of the problem (3) with singularities in $S_{3}$. We have to find a piecewise meromorphic solution of the problem (3), disappearing at infinity and with a given principal part $f^{\prime}(z)$ of the component $v_{3}(z)$.

For the sake of definiteness, let

$$
f^{\prime}(z)=F(z)=\sum_{j=1}^{m} \frac{c_{j}}{(z-E)^{j}} .
$$

For a convenience and simplification of the forthcoming evaluations we consider a conformal mapping of the $z$-plane onto the $\zeta$-plane by the help of the Möbius function

$$
\zeta(z)=T^{-1}(z)=\frac{z-a}{z-b},
$$

where $a$ and $b$ are the points symmetric about both circles $\mathcal{L}$ and $\ell$ simultaneously, i.e., $a b=r_{1}^{2},(a-h)(b-h)=r_{2}^{2}$, wherefrom it follows that

$$
a, b=\frac{h^{2}+r_{1}^{2}-r_{2}^{2}}{2 h} \mp \frac{\sqrt{\left(h^{2}-r_{1}^{2}-r_{2}^{2}\right)^{2}-4 r_{1}^{2} r_{2}^{2}}}{2 h} .
$$


The function (6) maps the eccentric annulus onto the concentric one, in particular, the domains $S_{j}, j=1,2,3$ onto the domains $S_{1}^{*}=\{\zeta:|\zeta|>R\}, S_{2}^{*}=\{\zeta: r<|\zeta|<R\}$, and $S_{3}^{*}=\{\zeta:|\zeta|<r\}$ correspondingly (Fig. 2), where

$$
R^{2}=\frac{a}{b}<1, \quad r^{2}=\frac{a-h}{b-h}<R^{2} .
$$

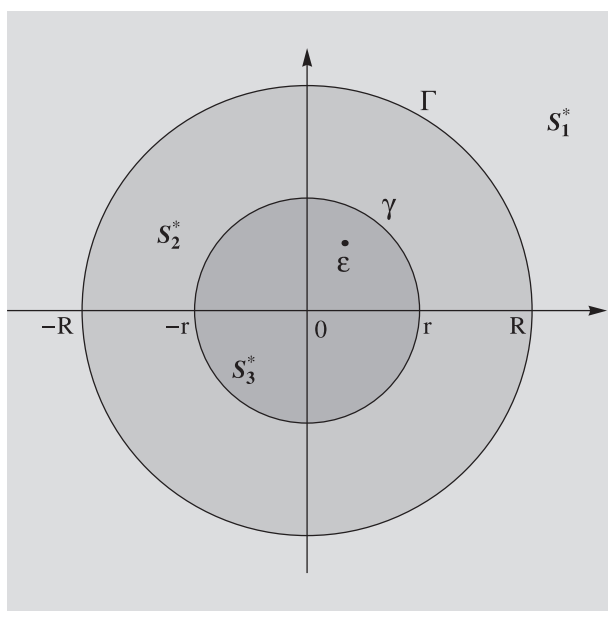

FIG. 2. Image of $z$-plane in $\zeta$-plane

We introduce a new unknown piecewise meromorphic function

$$
V(\zeta)=v[z(\zeta)]=V_{p}(\zeta), \quad \zeta \in S_{p}^{*}, \quad p=1,2,3,
$$

where

$$
z(\zeta)=T(\zeta)=\frac{b \zeta-a}{\zeta-1}
$$

is the inversion of the function (6).

Due to (3), (6), and (10), the function (9) satisfies the boundary conditions

$$
\begin{aligned}
& V_{2}(\tau)=A_{21} V_{1}(\tau)+B_{21} R^{2}\left[T_{1}(\tau)\right]^{-2} \overline{V_{1}(\tau)}, \quad \tau \in \Gamma=\{\tau:|\tau|=R\}, \\
& V_{2}(\tau)=A_{23} V_{3}(\tau)+B_{23} r^{2}\left[T_{2}(\tau)\right]^{-2} \overline{V_{3}(\tau)}, \quad \tau \in \gamma=\{\tau:|\tau|=r\},
\end{aligned}
$$

where

$$
T_{1}(\zeta)=\frac{\zeta-R^{2}}{\zeta-1}=T(\zeta) / b, \quad T_{2}(\zeta)=\frac{\zeta-r^{2}}{\zeta-1}=(T(\zeta)-h) /(b-h) .
$$

Due to (5), (9), and (10), a piecewise meromorphic solution of the boundary-value problem (11) has to have its principal part coinciding with

$$
Q(\zeta)=F[T(\zeta)]=\frac{c_{1}}{T_{3}(\zeta)}+\frac{Q_{0}(\zeta)}{\left[T_{3}(\zeta)\right]^{2}}
$$

where

$$
T_{3}(\zeta)=T(\zeta)-E=(b-E) \frac{\zeta-\mathcal{E}}{\zeta-1}, \quad \mathcal{E}=\frac{E-a}{E-b} .
$$


Let us represent the functions $V_{j}(\zeta)$ as follows:

$$
V_{1}(\zeta)=\frac{a_{1}}{T(\zeta)}+V_{10}(\zeta), V_{2}(\zeta)=V_{2}^{+}(\zeta)+V_{2}^{-}(\zeta), V_{3}(\zeta)=Q(\zeta)+V_{30}(\zeta)
$$

where a coefficient $a_{1}$ is to be found later,

$$
V_{1}(1)=V_{10}(1)=V_{10}^{\prime}(1)=0, \quad V_{2}^{-}(1)=Q(1)=0,
$$

and the functions $V_{10}(\zeta), V_{2}^{+}(\zeta), V_{2}^{-}(\zeta), V_{30}(\zeta)$ are holomorphic in the domains $S_{1}$, $S_{2} \cup \overline{S_{3}}, S_{2} \cup \overline{S_{1}}, S_{3}$ respectively.

We now consider the functions

$$
\begin{gathered}
\Phi(\zeta)= \begin{cases}V_{2}^{+}(\zeta)-B_{21}\left[R / T_{1}(\zeta)\right]^{2} \overline{V_{1}\left(R^{2} / \bar{\zeta}\right)}, & |\zeta|<R, \\
A_{21} V_{1}(\zeta)-V_{2}^{-}(\zeta), & |\zeta|>R,\end{cases} \\
\Psi(\zeta)= \begin{cases}V_{2}^{+}(\zeta)-A_{23} V_{30}(\zeta)-B_{23}\left[r / T_{2}(\zeta)\right]^{2} \overline{Q\left(r^{2} / \bar{\zeta}\right)}, & |\zeta|<r, \\
A_{23} Q(\zeta)-V_{2}^{-}(\zeta)+B_{23}\left[r / T_{2}(\zeta)\right]^{2} \overline{V_{30}\left(r^{2} / \bar{\zeta}\right)}, & |\zeta|>r .\end{cases}
\end{gathered}
$$

In accordance with the boundary conditions (11) the functions (17) and (18) have no jumps across the interfaces $\mathcal{L}$ and $\ell$ respectively. Hence, $\Phi(\zeta)(\Psi(\zeta))$ is holomorphic everywhere in $\mathbb{C}$, except at the point $\zeta=R^{2}\left(\zeta=r^{2}\right)$, where it has a simple pole. Besides, these functions vanish at the point $\zeta=1$ and are bounded at infinity. Due to the generalized Liouville theorem,

$$
\Phi(\zeta)=\alpha / T_{1}(\zeta), \quad \Psi(\zeta)=\beta / T_{2}(\zeta) .
$$

Both constants $\alpha$ and $\beta$ have to be determined from the holomorphicity condition of the functions $V_{2}^{+}(\zeta)$ and $V_{30}(\zeta)$ at the points $\zeta=R^{2}$ and $\zeta=r^{2}$. Namely, due to (15) through (17) we have

$$
V_{2}^{+}(\zeta)=\frac{\alpha}{T_{1}(\zeta)}+B_{21} \frac{R^{2}}{\left[T_{1}(\zeta)\right]^{2}}\left(\frac{\bar{a}_{1}}{T\left(R^{2} / \zeta\right)}+\overline{V_{10}\left(R^{2} / \bar{\zeta}\right)}\right)
$$

The last summand here is holomorphic at the point $\zeta=R^{2}$, but the sum of the first two will be holomorphic iff

$$
\alpha=-B_{21} R^{2} \bar{a}_{1} \lim _{\zeta \rightarrow R^{2}} 1 /\left[T_{1}(\zeta) T\left(R^{2} / \zeta\right)\right]
$$

taking into account that, due to (10),

$$
T\left(R^{2} / \zeta\right)=a / T_{1}(\zeta)
$$

Using representations (8), (12) it is not difficult to get

$$
\alpha=-B_{21} \bar{a}_{1} / b \text {. }
$$

From (8), (10), (12) through (18) it follows that

$$
\beta=-B_{23} r^{2} \bar{c}_{1} \lim _{\zeta \rightarrow r^{2}} 1 /\left[\overline{T_{3}\left(r^{2} / \bar{\zeta}\right)} T_{2}(\zeta)\right]=-B_{23} \bar{c}_{1} /(b-h) .
$$

Next, from (15), (17), (19), (21) after some simple algebra we get

$$
\begin{aligned}
& V_{2}^{+}(\zeta)-B_{21} R^{2}\left[T_{1}(\zeta)\right]^{-2} \overline{V_{10}\left(R^{2} / \bar{\zeta}\right)}=0, \\
& A_{21} \overline{V_{10}\left(R^{2} / \bar{\zeta}\right)}-\overline{V_{2}^{-}\left(R^{2} / \bar{\zeta}\right)}=-T_{1}(\zeta)\left(A_{21} \bar{a}_{1}+B_{21} a_{1}\right) / a, \quad|\zeta|<R .
\end{aligned}
$$


Exclusion of the function $V_{10}(\zeta)$ from the system (23) leads to the following relation:

$$
A_{21} V_{2}^{+}(\zeta)-\frac{B_{21} R^{2}}{\left[T_{1}(\zeta)\right]^{2}} \overline{V_{2}^{-}\left(R^{2} / \bar{\zeta}\right)}=-B_{21}\left(A_{21} \bar{a}_{1}+B_{21} a_{1}\right) / T(\zeta) .
$$

Analogous derivations, using (8), (12), (13), (18), (19), (22), result in

$$
\begin{aligned}
V_{2}^{+}(\zeta)-A_{23} V_{30}(\zeta) & -\frac{B_{23} r^{2}}{\left[L_{2}(\zeta)\right]^{2}} \overline{Q_{0}\left(r^{2} / \bar{\zeta}\right)}=\frac{B_{23} \bar{c}_{1}(\bar{E}-h)}{(b-h) L_{2}(\zeta)}, \\
B_{23} V_{30}(\zeta) & -\frac{r^{2}}{\left[T_{2}(\zeta)\right]^{2}} \overline{V_{2}^{-}\left(r^{2} / \bar{\zeta}\right)}+\frac{A_{23} r^{2}}{\left[L_{2}(\zeta)\right]^{2}} \overline{Q_{0}\left(r^{2} / \bar{\zeta}\right)} \\
& =-\frac{r^{2}}{T_{2}(\zeta)}\left(\frac{B_{23} c_{1}}{a-h}+\frac{A_{23} \bar{c}_{1}}{L_{2}(\zeta)}\right), \quad|\zeta|<r
\end{aligned}
$$

where

$$
L_{2}(\zeta)=(a-\bar{E}) \frac{\zeta-r^{2} / \overline{\mathcal{E}}}{\zeta-1} .
$$

Exclusion of the function $V_{30}(\zeta)$ from (25) gives

$$
\begin{aligned}
B_{32} V_{2}^{+}(\zeta)+\frac{A_{32} r^{2}}{\left[T_{2}(\zeta)\right]^{2}} \overline{V_{2}^{-}\left(r^{2} / \bar{\zeta}\right)}-\frac{r^{2}}{\left[L_{2}(\zeta)\right]^{2}} \overline{Q_{0}\left(r^{2} / \bar{\zeta}\right)} \\
\quad=\frac{\bar{c}_{1} r^{2}}{L_{2}(\zeta)}\left(\frac{B_{23} B_{32}(\bar{E}-h)}{a-h}+\frac{A_{23} A_{32}}{T_{2}(\zeta)}\right)-\frac{A_{23} B_{32} c_{1}}{(b-h) T_{2}(\zeta)} .
\end{aligned}
$$

Our aim at this stage is to get a functional equation about $V_{2}^{+}$by exclusion of the function $V_{2}^{-}$from the equations (24), (27). For this purpose we designate

$$
g=r^{2} / R^{2}
$$

and substitute $g \zeta$ instead of $\zeta$ into (27). Then the exclusion of $V_{2}^{-}$results in

$$
\begin{aligned}
V_{2}^{+}(\zeta)=\Delta_{21} & {\left[\frac{g \Delta_{23} V_{2}^{+}(g \zeta)}{[S(\zeta)]^{2}}+\left(1-\Delta_{23}\right) \frac{R^{2}}{\left[L_{1}(\zeta)\right]^{2}} \overline{Q_{0}\left(R^{2} / \bar{\zeta}\right)}\right.} \\
& \left.-\frac{B_{23}(\bar{E}-h) \Delta_{23} \bar{c}_{1}}{(b-h) S(\zeta) L_{1}(\zeta)}-\frac{1}{T(\zeta)}\left(A_{21} \bar{a}_{1}+B_{21} a_{1}-\frac{a A_{23} \bar{c}_{1}}{L_{1}(\zeta)}-\frac{B_{23} b c_{1}}{(b-h) S(\zeta)}\right)\right],
\end{aligned}
$$

where

$$
S(\zeta)=\frac{g \zeta-1}{\zeta-1}, \quad L_{1}(\zeta)=(a-\bar{E}) \frac{\zeta-R^{2} / \overline{\mathcal{E}}}{\zeta-1}
$$

In order to get the equation (29), the equations (10), (12), (14), (26), (30) are involved in the following relations:

$$
\begin{gathered}
\frac{T_{2}(g \zeta)}{T_{1}(\zeta)}=\frac{L_{2}(g \zeta)}{L_{1}(\zeta)}=\frac{g}{S(\zeta)}, \quad \overline{T_{3}\left(R^{2} / \bar{\zeta}\right)}=\frac{L_{1}(\zeta)}{T_{1}(\zeta)}, \quad \overline{T_{3}\left(r^{2} / \bar{\zeta}\right)}=\frac{L_{2}(\zeta)}{T_{2}(\zeta)} \\
\overline{T_{1}\left(R^{2} / \bar{\zeta}\right)}=\frac{R^{2}}{T_{1}(\zeta)}, \quad \overline{T_{2}\left(r^{2} / \bar{\zeta}\right)}=\frac{r^{2}}{T_{2}(\zeta)}, \quad L_{2}(g \zeta)=g \frac{L_{1}(\zeta)}{S(\zeta)}
\end{gathered}
$$

The left-hand side of the equation (29) is holomorphic at the point $\zeta=R^{2}$, but its right-hand side has there, generally speaking, a simple pole. This singularity vanishes if

$$
A_{21} \bar{a}_{1}+B_{21} a_{1}-\frac{a A_{23} \bar{c}_{1}}{L_{1}\left(R^{2}\right)}-\frac{B_{23} b c_{1}}{(b-h) S\left(R^{2}\right)}=0,
$$


wherefrom the coefficient $a_{1}$ in (15), due to (30) and (44), (8), (14), could be found as

$$
A_{21} \bar{a}_{1}+B_{21} a_{1}=A_{23} \bar{c}_{1}+B_{23} c_{1} \text { and } a_{1}=A_{13} c_{1}+B_{13} \bar{c}_{1} .
$$

Using (32) and the equality

$$
\frac{1}{S(\zeta) L_{1}(\zeta)}=\frac{b-h}{h-\bar{E}}\left(\frac{h /[a(b-h)]}{S(\zeta)}-\frac{\bar{E} / b}{L_{1}(\zeta)}\right),
$$

the functional equation (29) could be transformed to the following one:

$$
V_{2}^{+}(\zeta)=\delta g \frac{V_{2}^{+}(g \zeta)}{[S(\zeta)]^{2}}+C_{1} \frac{\overline{Q_{0}\left(R^{2} / \bar{\zeta}\right)}}{\left[L_{1}(\zeta)\right]^{2}}+\frac{C_{2}}{L_{1}(\zeta)}+\frac{C_{3}}{S(\zeta)},
$$

where

$$
\begin{gathered}
\delta=\Delta_{21} \Delta_{23}, \quad|\delta|<1 \\
C_{1}=\Delta_{21}\left(1-\Delta_{23}\right) R^{2}, C_{2}=\bar{E} C_{1} \bar{c}_{1} / a, C_{3}=\frac{\delta h\left(c_{1}+\Delta_{23} \bar{c}_{1}\right)}{\left(1+\Delta_{23}\right) a(b-h)} .
\end{gathered}
$$

We introduce two sets of Möbius transformations:

$$
S_{k}(\zeta)=\frac{g^{k} \zeta-1}{\zeta-1}, \quad \Lambda_{k}(\zeta)=(a-\bar{E}) \frac{g^{k} \zeta-R^{2} / \overline{\mathcal{E}}}{\zeta-1}, \quad k=0,1, \ldots
$$

From definitions (30), (36) we have

$$
\begin{gathered}
S_{0}(\zeta) \equiv 1, \quad S_{1}(\zeta)=S(\zeta), \quad \Lambda_{0}(\zeta)=L_{1}(\zeta) \\
S_{1}\left(g^{k} \zeta\right) S_{k}(\zeta)=S_{k+1}(\zeta), \quad S_{k}(\zeta) \Lambda_{0}\left(g^{k} \zeta\right)=\Lambda_{k}(\zeta)
\end{gathered}
$$

In order to solve the equation (33), we change there $\zeta$ for $g \zeta$ and substitute the result into the right-hand part of (33). Thus we get

$$
\begin{aligned}
V_{2}^{+}(\zeta)=(\delta g)^{2} \frac{V_{2}^{+}\left(g^{2} \zeta\right)}{\left[S_{2}(\zeta)\right]^{2}} & +C_{1}\left(\frac{\overline{Q_{0}\left(R^{2} / \bar{\zeta}\right)}}{\left[\Lambda_{0}(\zeta)\right]^{2}}+\frac{\overline{Q_{0}\left(R^{2} g^{-1} / \bar{\zeta}\right)}}{\left[\Lambda_{1}(\zeta)\right]^{2}}\right) \\
& +C_{2}\left(\frac{1}{\Lambda_{0}(\zeta)}+\frac{\delta g}{S_{1}(\zeta) \Lambda_{1}(\zeta)}\right)+C_{3}\left(\frac{1}{S_{1}(\zeta)}+\frac{\delta g}{S_{1}(\zeta) S_{2}(\zeta)}\right)
\end{aligned}
$$

Next, we substitute into the right-hand part of the last equation the result of change of $\zeta$ by $g^{2} \zeta$ in (33) and so on. We continue this procedure and at the $n$-th step we obtain

$$
V_{2}^{+}(\zeta)=(\delta g)^{n} \frac{V_{2}^{+}\left(g^{n} \zeta\right)}{\left[S_{n}(\zeta)\right]^{2}}+\sum_{k=0}^{n-1}(\delta g)^{k}\left(\frac{C_{1} \overline{Q_{0}\left(R^{2} g^{-k} / \bar{\zeta}\right)}}{\left[\Lambda_{k}(\zeta)\right]^{2}}+\frac{C_{2}}{S_{k}(\zeta) \Lambda_{k}(\zeta)}+\frac{C_{3}}{S_{k}(\zeta) S_{k+1}(\zeta)}\right) .
$$

In the limit when $n \rightarrow \infty$ the last formula gives the following solution of the equation (33):

$$
V_{2}^{+}(\zeta)=\sum_{k=0}^{\infty}(\delta g)^{k}\left(\frac{C_{1} \overline{Q_{0}\left(R^{2} g^{-k} / \bar{\zeta}\right)}}{\left[\Lambda_{k}(\zeta)\right]^{2}}+\frac{C_{2}}{S_{k}(\zeta) \Lambda_{k}(\zeta)}+\frac{C_{3}}{S_{k}(\zeta) S_{k+1}(\zeta)}\right)
$$

The last series converges absolutely and uniformly for $|\zeta|<R$, as $|\delta g|<1$, and

$$
\begin{aligned}
\min \left|S_{k}(\zeta)\right| & >\frac{1-R}{1+R}>0, \quad \min \left|\Lambda_{k}(\zeta)\right|>\frac{|b-E| R^{2}-|a-E| R}{1+R}>0, \\
& \min \left|T_{3}\left(R^{2} g^{-k} / \bar{\zeta}\right)\right|>\frac{|b-E| R-|a-E|}{1+R}>0 .
\end{aligned}
$$


From relations (15), (23), (24), (25), (32) and the corresponding equalities (31) successively follows

$$
\begin{gathered}
V_{1}(\zeta)=\frac{A_{13} c_{1}+B_{13} \bar{c}_{1}}{T(\zeta)}+\frac{r_{1}^{2}}{B_{21}[T(\zeta)]^{2}} \overline{V_{2}^{+}\left(R^{2} / \bar{\zeta}\right)} \\
V_{2}^{-}(\zeta)=\frac{r_{1}^{2}}{\Delta_{21}[T(\zeta)]^{2}} \overline{V_{2}^{+}\left(R^{2} / \bar{\zeta}\right)}+\left(A_{23} c_{1}+B_{23} \bar{c}_{1}\right) / T(\zeta), \\
V_{30}(\zeta)=\left(1+\Delta_{23}\right) V_{2}^{+}(\zeta)-\frac{\Delta_{23} r^{2}}{\left[L_{2}(\zeta)\right]^{2}} \overline{Q_{0}\left(r^{2} / \bar{\zeta}\right)}-\frac{\Delta_{23} \bar{c}_{1}(\bar{E}-h)}{(b-h) L_{2}(\zeta)} .
\end{gathered}
$$

A solution of the problem (11) is now determined by the formulae (15). Then, the corresponding solution of the initial problem (3) could be found in accordance with (9).

First, we list the results of changing $\zeta$ with $\zeta(z)$ by (6) in (14), (26) , (36). Omitting tiresome algebra we write down the final results of the derivations:

$$
\begin{gathered}
\overline{T_{3}\left(R^{2} g^{-k} / \bar{\zeta}\right)}=\left(z_{-k}-\bar{E}\right) \frac{z-E_{k}^{*}}{z-z_{-k}}, \quad T_{3}\left(g^{-k} \zeta\right)=\left(z_{k}^{*}-E\right) \frac{z-E_{k}}{z-z_{-k}^{*}}, \\
L_{2}(\zeta)=\frac{h-\bar{E}}{b-h}\left(z-E^{*}\right), \quad E^{*}=T\left(r^{2} / \overline{\mathcal{E}}\right)=h+\frac{r_{2}^{2}}{\bar{E}-h}, \\
S_{k}(\zeta)=-\lambda_{k}\left(z-z_{k}^{*}\right), \quad \overline{S_{k}\left(R^{2} / \bar{\zeta}\right)}=\lambda_{k} z_{k}^{*} \frac{z-z_{k}}{z}, \\
\Lambda_{0}(\zeta)=-\frac{\bar{E}}{b}\left(z-E_{0}^{*}\right), \quad \Lambda_{k}(\zeta)=a \lambda_{k} \frac{\bar{E}-z_{-k}}{z_{-k}}\left(z-E_{k}^{*}\right), \quad k \in \mathbb{N}, \\
\overline{\Lambda_{0}\left(R^{2} / \bar{\zeta}\right)}=a \frac{z-E}{z}, \quad \overline{\Lambda_{k}\left(R^{2} / \bar{\zeta}\right)}=a \lambda_{k}\left(z_{k}^{*}-E\right) \frac{z-E_{k}}{z}, \quad k \in \mathbb{N}, \\
\overline{T_{3}\left(r^{2} / \bar{\zeta}\right)}=(h-\bar{E}) \frac{z-E^{*}}{z-h},
\end{gathered}
$$

where $\lambda_{k}=\left(1-g^{k}\right) /(b-a)$,

$$
\begin{aligned}
z_{k} & =r_{1}^{2} / T\left(g^{-k}\right), \quad z_{k}^{*}=T\left(g^{-k}\right), \quad k=0, \pm 1, \pm 2, \ldots . \\
E_{k} & =T\left(g^{k} \mathcal{E}\right), \quad E_{k}^{*}=r_{1}^{2} / T\left(g^{k} \overline{\mathcal{E}}\right),
\end{aligned}
$$

Wherefrom, in particular, follows: $z_{0}=0, z_{0}^{*}=\infty, E_{0}=E, E_{0}^{*}=r_{1}^{2} / \bar{E}, z_{1}=h$, $z_{1}^{*}=r_{1}^{2} / h$.

Note some properties of introduced consequences $\left\{z_{ \pm k}\right\},\left\{z_{ \pm k}^{*}\right\},\left\{E_{ \pm k}\right\},\left\{E_{ \pm k}^{*}\right\}$ :

First, it is evident that points $z_{k}$ and $z_{k}^{*}, E_{k}$ and $E_{k}^{*}$ are symmetric about the circumference $\mathcal{L}$. Less evident is the symmetry of the pairs $\left\{z_{k+1}, z_{k}^{*}\right\}$ and $\left\{E_{k+1}, E_{k}^{*}\right\}$ about the circumference $\ell$, i.e.

$$
h+\frac{r_{2}^{2}}{z_{k}^{*}-h}=z_{k+1}, \quad h+\frac{r_{2}^{2}}{\overline{E_{k}^{*}}-h}=E_{k+1}, \quad k=0, \pm 1, \pm 2, \ldots .
$$

Second, all sequences are converging. Namely, if $k \rightarrow \infty$, then $\left\{z_{k}\right\} \rightarrow a\left(\left\{z_{-k}\right\} \rightarrow b\right)$, $\left\{z_{k}^{*}\right\} \rightarrow b\left(\left\{z_{-k}^{*}\right\} \rightarrow a\right),\left\{E_{k}\right\} \rightarrow a,\left(\left\{E_{-k}\right\} \rightarrow b\right),\left\{E_{k}^{*}\right\} \rightarrow b\left(\left\{E_{-k}^{*}\right\} \rightarrow a\right)$.

Third, the set of points $\left\{g^{k} \mathcal{E}\right\}, k=0, \pm 1, \pm 2, \ldots$ belongs to the straight line going through the origin and point $\mathcal{E}=T(E)$. The Möbius transformation $T(\zeta)$ maps this straight line onto the circle orthogonal with both circles $\mathcal{L}$ and $\ell$ and going through the points $a, b, E$, and $E_{0}^{*}$. Hence all points of both consequences $\left\{E_{k}\right\}$ and $\left\{E_{k}^{*}\right\}$ are situated in the last circle in accordance with (44). 
Fourth, the following relations are useful for the forthcoming evaluations:

$$
\begin{aligned}
& \frac{z_{-k} \bar{E}\left(r_{1} \lambda_{k}\right)^{-2}}{\left(E_{k}^{*}-z_{k}^{*}\right)\left(\bar{E}-z_{-k}\right)}=\frac{\left(z_{-k} /\left(r_{1} \lambda_{k}\right)\right)^{2}}{\left(E_{k}^{*}-z_{-k}\right)\left(\bar{E}-z_{-k}\right)}=g^{-k}, \\
& \frac{z_{k} E\left(r_{1} \lambda_{k}\right)^{-2}}{\left(E_{k}-z_{k}\right)\left(z_{k}^{*}-E\right)}=\frac{\lambda_{k}^{-2}}{\left(E_{k}-z_{-k}^{*}\right)\left(z_{k}^{*}-E\right)}=g^{-k},
\end{aligned}
$$

where $g$ is given by (30).

Fifth and the last, it is worth pointing out that

$$
z_{k}^{*}+z_{-k}^{*}=a+b, \quad 1 / z_{k}+1 / z_{-k}=1 / a+1 / b, \quad k=1,2, \ldots .
$$

It is convenient to represent the derivative of the given complex potential (5) as follows:

$$
F(z)=\frac{c_{1}}{z-E}+\frac{1}{z-E} \sum_{j=2}^{m} \frac{c_{j}}{(z-E)^{j-1}}=\frac{1}{z-E}\left(c_{1}+F_{0}(z)\right) .
$$

The required solution of the initial problem (3) could be presented now in accordance with formulae (15), (13), (9), (6) and relations (39) through (46) in the following form:

$$
\begin{aligned}
& v_{1}(z)=\frac{\Delta_{12}\left(\bar{c}_{1}+\Delta_{13} c_{1}\right)}{\left(1+\Delta_{13}\right) z}+\left(1+\Delta_{21}\right)\left(1-\Delta_{23}\right)\left(\omega_{1}(z)-\Omega_{1}(z)\right), \\
& v_{2}(z)=\left(1-\Delta_{23}\right)\left[\omega_{1}(z)-\Omega_{1}(z)-\Delta_{21}\left(\omega_{2}(z)+\mathbf{S}_{1} \Omega_{1}(z)\right)\right], \\
& v_{3}(z)=F(z)+\Delta_{23}\left[\frac{\bar{c}_{1}}{z-h}-\mathbf{S}_{2} F(z)\right]-\Delta_{21}\left(1-\Delta_{23}^{2}\right)\left(\omega_{2}(z)+\mathbf{S}_{1} \Omega_{1}(z)\right),
\end{aligned}
$$

where the following two operators are used:

$$
\mathbf{S}_{1} \phi(z)=\frac{r_{1}^{2}}{z^{2}} \overline{\phi\left(\frac{r_{1}^{2}}{\bar{z}}\right)}, \quad \mathbf{S}_{2} \phi(z)=\frac{r_{2}^{2}}{(z-h)^{2}} \overline{\phi\left(h+\frac{r_{2}^{2}}{\bar{z}-h}\right)},
$$

and

$$
\begin{gathered}
\omega_{1}(z)=\sum_{k=0}^{\infty} \delta^{k}\left(\frac{c_{1}}{z-E_{k}}+\frac{\Delta_{23}\left(1-\Delta_{21}\right)}{\left(1-\Delta_{23}\right)\left(1+\Delta_{13}\right)} \frac{\left(\bar{c}_{1}+\Delta_{13} c_{1}\right)}{z-z_{k+1}}\right), \\
\omega_{2}(z)=\sum_{k=0}^{\infty} \delta^{k}\left(\frac{\bar{c}_{1}}{z-E_{k}^{*}}+\frac{\Delta_{23}\left(1-\Delta_{21}\right)}{\left(1-\Delta_{23}\right)\left(1+\Delta_{13}\right)} \frac{\left(c_{1}+\Delta_{13} \bar{c}_{1}\right)}{z-z_{k+1}^{*}}\right), \\
\Omega_{1}(z)=\sum_{k=0}^{\infty} \delta^{k}\left(\frac{1}{z-z_{-k}^{*}}-\frac{1}{z-E_{k}}\right) F_{0}\left(z_{k}^{*} \frac{z-z_{k}}{z-z_{-k}^{*}}\right) .
\end{gathered}
$$

In its turn,

$$
F_{0}\left(z_{k}^{*} \frac{z-z_{k}}{z-z_{-k}^{*}}\right)=\sum_{j=2}^{m} c_{j}\left[\frac{z-z_{-k}^{*}}{\left(z_{k}^{*}-E\right)\left(z-E_{k}\right)}\right]^{j-1} .
$$

REMARK 1. It could be shown that the following identities hold:

$$
\begin{gathered}
\mathbf{S}_{1} \omega_{1}(z) \equiv-\omega_{2}(z)+\frac{\left(\bar{c}_{1}+\Delta_{23} c_{1}\right)}{\left(1-\Delta_{23}^{2}\right) z}, \quad \mathbf{S}_{2} \mathbf{S}_{1} \Omega_{1}(z) \equiv \frac{1}{\delta}\left(\Omega_{1}(z)+\frac{F_{0}(z)}{z-E}\right), \\
\mathbf{S}_{2} \omega_{2}(z) \equiv \frac{1}{\delta}\left(\frac{c_{1}}{z-E}-\omega_{1}(z)\right)+\frac{\bar{c}_{1}+\Delta_{23} c_{1}}{\Delta_{21}\left(1-\Delta_{23}^{2}\right)(z-h)} .
\end{gathered}
$$


Using relations (52) one can easily ascertain that the functions (47) satisfy the boundary conditions (3). To prove this it is convenient to rewrite (3), using the operators (48), in the following equivalent form:

$$
\begin{aligned}
\left(1+\Delta_{21}\right) v_{2}(t) & =v_{1}(t)+\Delta_{21} \mathbf{S}_{1} v_{1}(t), \quad t \in \mathcal{L}, \\
\left(1+\Delta_{23}\right) v_{2}(t) & =v_{3}(t)+\Delta_{23} \mathbf{S}_{2} v_{3}(t), \quad t \in \ell .
\end{aligned}
$$

REMARK 2. By integrating the solution (47), the corresponding disturbed complex potential could be written down as follows:

$$
\begin{aligned}
& w_{1}(z)=\left(1+\Delta_{21}\right) \sigma_{1}(z)-\frac{\Delta_{21}\left(\bar{c}_{1}+\Delta_{13} c_{1}\right)}{\left(1+\Delta_{13}\right)} \ln z \\
& \left.w_{2}(z)=\sigma_{1}(z)-\Delta_{21} \sigma_{2}(z), \quad \overline{\left(h+\frac{r_{2}^{2}}{\bar{z}-h}\right)}\right]-\Delta_{21}\left(1+\Delta_{23}\right) \sigma_{2}(z), \\
& w_{3}(z)=f(z)+\Delta_{23}\left[\bar{c}_{1} \ln (z-h)+f\right)
\end{aligned}
$$

where $f(z)$ is the given complex potential:

$$
\begin{gathered}
f(z)=c_{1} \ln (z-E)+\sum_{j=2}^{m} \frac{c_{j}}{1-j}(z-E)^{1-j} \\
\sigma_{1}(z)=\left(1-\Delta_{23}\right) \sum_{k=0}^{\infty} \delta^{k}\left[f\left(z_{k}^{*} \frac{z-z_{k}}{z-z_{-k}^{*}}\right)+c_{1} \delta \ln \left(z-z_{-k-1}^{*}\right)\right] \\
+\frac{\Delta_{23}\left(1-\Delta_{21}\right)\left(\bar{c}_{1}+\Delta_{13} c_{1}\right)}{1+\Delta_{13}} \sum_{k=0}^{\infty} \delta^{k} \ln \left(z-z_{k+1}\right), \\
\sigma_{2}(z)=\left(1-\Delta_{23}\right) \sum_{k=0}^{\infty} \delta^{k}\left[f\left(z_{-k} \frac{\bar{z}-z_{k}^{*}}{\bar{z}-z_{-k}}\right)\right. \\
\left.+\frac{\Delta_{23}\left(1-\Delta_{21}\right)\left(c_{1}+\Delta_{13} \bar{c}_{1}\right)}{1+\Delta_{13}} \sum_{k=0}^{\infty} \ln \left(z-z_{-k}\right)\right]
\end{gathered}
$$

The principal values of all logarithmic functions $\ln (z-\tau)$ involved in (53) through (55) are fixed in the $z$-plane with the cut along the ray $\{z: \arg (z-\tau)=\pi\}$.

ExAmple 1. Let $r_{1}=4, r_{2}=2, h=1, \rho_{1}=1, \rho_{2}=0.2, \rho_{3}=2, E=1.5+0.5 \mathrm{i}$. In Fig. 3 the streamlines and equipotential lines (dashed) are plotted for two cases: a) the source at the point $E\left(m=1, c_{1}=1+0.1 \mathrm{i}\right)$ due to the formulae (47) through (51) and b) the dipole $\left(m=2, c_{1}=0, c_{2}=1-\mathrm{i}\right)$ in accordance with formulae (553) through (55)).

\section{Solution of the problem (3) with singularities $F(z)$ within the annulus} $S_{2}$. Let $F(z)$ be defined as above by (5) but now $E \in S_{2}$. The corresponding solution of the problem (3) can be found similarly to the previous section. We get here the same problem (11) about a function (9) in an auxiliary $\zeta$-plane. If we represent

$$
V_{1}(\zeta)=\frac{a_{1}}{T(\zeta)}+V_{10}(\zeta), V_{2}(\zeta)=Q(\zeta)+V_{2}^{+}(\zeta)+V_{2}^{-}(\zeta)
$$

then, using conditions (16), it is not difficult to show that

$$
\frac{\alpha}{T_{1}(\zeta)}= \begin{cases}V_{2}^{+}(\zeta)-B_{21}\left[R / T_{1}(\zeta)\right]^{2} \overline{V_{1}\left(R^{2} / \bar{\zeta}\right)}, & |\zeta|<R \\ A_{21} V_{1}(\zeta)-V_{2}^{-}(\zeta)-Q(\zeta), & |\zeta|>R .\end{cases}
$$



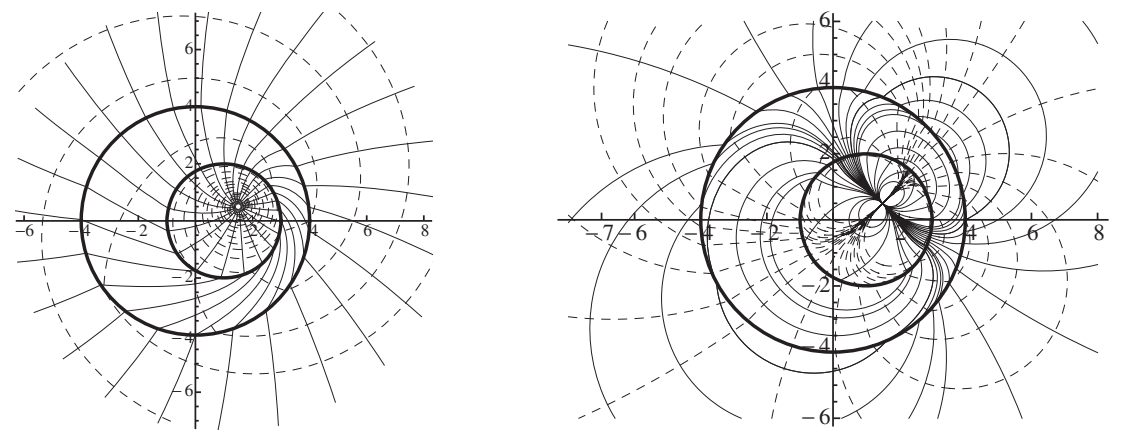

FIG. 3. The case of a source (left panel) and a dipole (right panel) inside $S_{3}$.

$$
0= \begin{cases}V_{2}^{+}(\zeta)+Q(\zeta)-A_{23} V_{30}(\zeta), & |\zeta|<r, \\ -V_{2}^{-}(\zeta)+B_{23}\left[r / T_{2}(\zeta)\right]^{2} V_{3}\left(r^{2} / \bar{\zeta}\right), & |\zeta|>r .\end{cases}
$$

From the last equalities one can find successively $\alpha=-B_{21} \bar{a}_{1} / b, a_{1}=A_{12} c_{1}+B_{12} \bar{c}_{1}$ and the following functional equation about $V_{2}^{+}(\zeta)$ :

$$
V_{2}^{+}(\zeta)=\delta g \frac{V_{2}^{+}(g \zeta)+Q(g \zeta)}{[S(\zeta)]^{2}}+\Delta_{21}\left[\frac{\overline{R^{2} Q_{0}\left(R^{2} / \bar{\zeta}\right)}}{\left[L_{1}(\zeta)\right]^{2}}+\frac{\bar{c}_{1} \bar{E}}{b L_{1}(\zeta)}\right]
$$

Using (30), (31), (36), (37) we derive first a solution $V_{2}^{+}(\zeta)$ of the last equation and then all other components of the function $V(\zeta)$. The inverse change $\zeta$ with $\zeta(z)$ by (6) allows us to write down the required solution of the initial problem (3) in the following form:

$$
\begin{aligned}
v_{1}(z)=\left(1+\Delta_{21}\right) & {\left[F(z)-\Omega_{1}(z)+\frac{\Omega_{2}(z)+\omega_{2}(z)}{\Delta_{21}}\right]-\frac{\Delta_{21}\left(\bar{c}_{1}-\Delta_{21} c_{1}\right)}{\left(1-\Delta_{21}\right) z}, } \\
v_{2}(z)=F(z)- & \Omega_{1}(z)+\frac{\Omega_{2}(z)+\omega_{2}(z)}{\Delta_{21}} \\
+\Delta_{21} \mathbf{S}_{1}\left(F(z)-\Omega_{1}(z)-\frac{c_{1}}{z}\right)+\mathbf{S}_{1} \Omega_{2}(z)-\omega_{1}(z), & \left.\left.c_{1}\right)+\mathbf{S}_{1} \Omega_{2}(z)-\omega_{1}(z)\right], \\
v_{3}(z)=\left(1+\Delta_{23}\right) & {\left[F(z)+\Delta_{21} \mathbf{S}_{1}\left(F(z)-\Omega_{1}(z)-\frac{c_{1}}{z}\right)\right.}
\end{aligned}
$$

where the first of two operators (48) is used and

$$
\begin{gathered}
\omega_{1}(z)=\sum_{k=1}^{\infty} \delta^{k}\left(\frac{c_{1}-\Delta_{21} \bar{c}_{1}}{z-z_{k}^{*}}+\frac{\Delta_{21} \bar{c}_{1}}{z-E_{k}^{*}}-\frac{c_{1}}{z-E_{-k}}\right), \\
\omega_{2}(z)=-\mathbf{S}_{1} \omega_{1}(z)=\sum_{k=1}^{\infty} \delta^{k}\left(\frac{\bar{c}_{1}-\Delta_{21} c_{1}}{z-z_{k}}+\frac{\Delta_{21} c_{1}}{z-E_{k}}-\frac{\bar{c}_{1}}{z-E_{-k}^{*}}\right),
\end{gathered}
$$

$\Omega_{1}(z)$ is defined in (50),

$$
\Omega_{2}(z)=\sum_{k=1}^{\infty} \delta^{k}\left(\frac{1}{z-z_{k}}-\frac{1}{z-E_{-k}^{*}}\right) \overline{F_{0}\left(z_{k} \frac{\bar{z}-z_{-k}^{*}}{\bar{z}-z_{k}}\right)},
$$

and points $z_{k}, z_{k}^{*}, E_{k}, E_{k}^{*}$ are given by (44). 
The functions (57) and (58) satisfy the identities

$$
\begin{gathered}
\mathbf{S}_{2} \mathbf{S}_{1}\left(F(z)-\Omega_{1}(z)-c_{1} / z\right) \equiv-\frac{\Omega_{1}(z)}{\delta}-c_{1}\left(\frac{1}{z-h}-\frac{1}{z-E_{1}}\right), \\
\mathbf{S}_{2} \mathbf{S}_{1} \Omega_{2}(z) \equiv \frac{\Omega_{2}(z)}{\delta}-\mathbf{S}_{2} F(z)+\bar{c}_{1}\left(\frac{1}{z-h}-\frac{1}{z-E^{*}}\right), \\
\mathbf{S}_{2} \omega_{1}(z) \equiv-\frac{\omega_{2}(z)}{\delta}-\frac{\bar{c}_{1}-\Delta_{21} c_{1}}{z-h}+\frac{\bar{c}_{1}}{z-E^{*}}-\frac{\Delta_{2} c_{1}}{z-E_{1}} .
\end{gathered}
$$

By the help of identities (59) it is quite easy to verify that the functions (56) satisfy the boundary conditions (3).

As well as in the previous case the integration of the solution (56) gives the corresponding complex potential:

$$
\begin{aligned}
& w_{1}(z)=\left(1+\Delta_{21}\right)(f(z)+\sigma(z))-\frac{\Delta_{21}\left(\bar{c}_{1}-\Delta_{21} c_{1}\right)}{\left(1-\Delta_{21}\right)} \ln \left(z / r_{1}\right), \\
& w_{2}(z)=f(z)+\sigma(z)-\Delta_{21}\left[\overline{f\left(r_{1}^{2} / \bar{z}\right)}+\overline{\sigma\left(r_{1}^{2} / \bar{z}\right)}+\bar{c}_{1} \ln \left(z / r_{1}\right)\right], \\
& w_{3}(z)=\left(1+\Delta_{23}\right)\left[f(z)-\Delta_{21}\left(\overline{f\left(r_{1}^{2} / \bar{z}\right)}+\overline{\sigma\left(r_{1}^{2} / \bar{z}\right)}+\bar{c}_{1} \ln \left(z / r_{1}\right)\right)\right],
\end{aligned}
$$

where $f(z)$ is the given complex potential (54) and

$$
\sigma(z)=\sum_{k=0}^{\infty} \delta^{k}\left[f\left(z_{k}^{*} \frac{z-z_{k}}{z-z_{-k}^{*}}\right)-\overline{f\left(z_{k} \frac{\bar{z}-z_{-k}^{*}}{\bar{z}-z_{k}}\right)} / \Delta_{21}+c_{1} \ln \frac{z_{k}\left(z-z_{-k}^{*}\right)}{r_{1}\left(z-z_{k}\right)}\right] .
$$
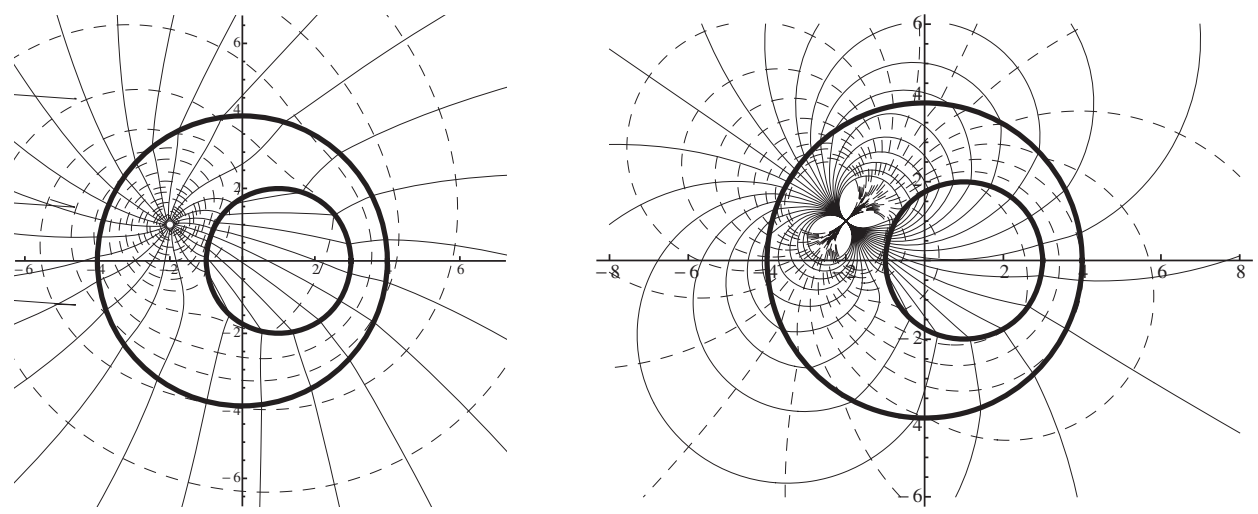

FIG. 4. The case of a source (left panel) and a dipole (right panel) at the point $E=-2+\mathrm{i}$ inside $S_{2}$

Example 2. In Fig. 4 the streamlines and equipotential lines are plotted due to the formulae (56) through (58) and (60), (61) for the same corresponding dates as in Example 1.

5. Solution of the problem (3) with singularities $F(z)$ within matrix $S_{1}$. The case when $E \in S_{1}$ in (5) is the most simple one. The required solution of the problem 
(3) has the following form:

$$
\begin{aligned}
& v_{1}(z)=F(z)+\left(1-\Delta_{21}^{2}\right) \Omega(z) / \Delta_{21}-\Delta_{21} \mathbf{S}_{1} F(z), \\
& v_{2}(z)=\left(1-\Delta_{21}\right)\left(F(z)+\Omega(z) / \Delta_{21}+\mathbf{S}_{1} \Omega(z)\right), \\
& v_{3}(z)=\left(1-\Delta_{21}\right)\left(1+\Delta_{23}\right)\left(F(z)+\mathbf{S}_{1} \Omega(z)\right),
\end{aligned}
$$

where

$$
\Omega(z)=\sum_{k=1}^{\infty} \delta^{k}\left(\frac{1}{z-z_{k}}-\frac{1}{z-E_{-k}^{*}}\right) \sum_{j=1}^{m} \bar{c}_{j}\left(\frac{z-z_{k}}{\left(z_{k}-\bar{E}\right)\left(z-E_{-k}^{*}\right)}\right)^{j-1} .
$$

Using the identity

$$
\mathbf{S}_{2} \mathbf{S}_{1} \Omega(z) \equiv \Omega(z) / \delta-\mathbf{S}_{2} F(z),
$$

one can easily check that the functions (62) satisfy the boundary conditions (3).

It could be shown that in accordance with (54), (64) the following representations are true:

$$
\begin{aligned}
\Omega(z) & =-\sum_{k=1}^{\infty} \delta^{k} \frac{\mathrm{d} \overline{f\left(z_{k}\left(\bar{z}-z_{-k}^{*}\right) /\left(\bar{z}-z_{k}\right)\right)}}{\mathrm{d} z}, \\
\mathbf{S}_{1} \Omega(z) & =-\sum_{k=1}^{\infty} \delta^{k} \frac{\mathrm{d} f\left(z_{-k}^{*}\left(z-z_{-k}\right) /\left(z-z_{k}^{*}\right)\right)}{\mathrm{d} z} .
\end{aligned}
$$

The complex potential, corresponding to the solution (62), has, due to (65), the form

$$
\begin{aligned}
& w_{1}(z)=f(z)+\Delta_{21} \overline{f\left(r_{1}^{2} / \bar{z}\right)}-\left(1 / \Delta_{21}-\Delta_{21}\right) \sigma(z), \\
& w_{2}(z)=\left(1-\Delta_{21}\right)\left[f(z)-\Delta_{21}^{-1} \sigma(z)+\overline{\sigma\left(r_{1}^{2} / \bar{z}\right)}\right] \\
& w_{3}(z)=\left(1+\Delta_{23}\right)\left(1-\Delta_{21}\right)\left[f(z)+\overline{\sigma\left(r_{1}^{2} / \bar{z}\right)}\right]
\end{aligned}
$$

where

$$
\sigma(z)=\sum_{k=1}^{\infty} \delta^{k} \overline{\left(z_{k} \frac{\bar{z}-z_{-k}^{*}}{\bar{z}-z_{k}}\right)}, \quad \overline{\sigma\left(r_{1}^{2} / \bar{z}\right)}=\sum_{k=1}^{\infty} \delta^{k} f\left(z_{-k}^{*} \frac{z-z_{-k}}{z-z_{k}^{*}}\right) .
$$

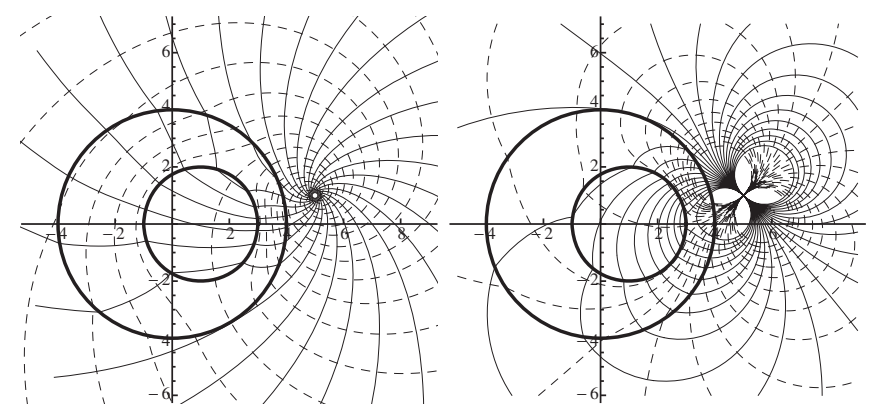

Fig. 5. The case of a source (on the left) and a dipole (on the right) at the point $E=5+\mathrm{i}$ inside $S_{1}$

ExAmple 3. The given complex potential $f(z)$ has a source (dipole) at the point $E=5+\mathrm{i}$ inside $S_{1}$ and $\rho_{1}=1, \rho_{2}=2, \rho_{3}=0.2, m=1, c_{1}=1+0.5 \mathrm{i}(m=2$, $\left.c_{1}=0, c_{2}=1-\mathrm{i}\right)$. The corresponding distributions of stream- and equipotential lines are represented in Fig. 5 
6. Conclusion and perspectives. We have analytically solved the problem of determination of a disturbed complex potential in an infinite matrix with a two-component eccentric annulus as an inclusion into a homogeneous matrix. Our solutions are derived for the cases when a given complex potential has an arbitrary singular point strictly inside one of three homogeneous zones. Owing to the linearity of the Laplace equation for a general case of a given potential with a finite number of singularities one can easily sum the corresponding solutions (47), (56), and (62). This principle of superposition allows us to consider practical scenarios in subsurface mechanics when a pair or an array of injection-abstraction wells is operated in a formation damaged in the vicinity of one well. In Obnosov et al. (2010) a special case has been studied when the production well had a two-phase skin with concentric circles as interfaces. The solution from the current paper will give the most general situation with an azimuthally arbitrary skin and arbitrary composition of wells in its vicinity.

We did not study the degenerating case of touching circles $\ell$ and $\mathcal{L}\left(h=r_{1}-r_{2}\right)$ as well as the case of singular points at the interface. As for the first of these cases, a required solution could be found via the formulae (47), (56), (62) as a corresponding limit when $l=r_{1}-r_{2}-h \rightarrow 0$. The case of boundary singularities was considered in [12] for a concentric annular inclusion. Detailed computations carried out by the help of the formulae obtained in this paper showed that if a singular point of $f(z)$ is very close to the interface, there is practically no difference in the flow net, compared with one for the case of the same singularity located exactly on the interface.

The method of solution utilized here was successively implemented for analytical investigations of many other $\mathbb{R}$-linear conjugation problems describing 2-D fields in groundwater hydrology, electrostatics, theory of elasticity, heat conduction and other applications where harmonic potentials emerge ([19]).

Acknowledgment. This work was supported by His Majesty Research Trust Fund, Oman, grant SR/AGR/SWAE/09/01, "Feasibility of Managed Aquifer Recharge Using Excess Treated Wastewater in Oman", as well as RFBR grants No 09-01-97008r_povolgh'e_a and No 09-01-12188-ofi_m. Financial support of the Russian Federal Agency of Science and Innovations (contract No 02.740.11.0193) is also appreciated. The comments of an anonymous referee were very helpful.

\section{REFERENCES}

[1] Crowdy D.G., Analytical solutions for uniform potential flow past multiple cylinders. European Journal of Mechanics, B/Fluids 25 (4), 459-470 (2006) MR2241382 (2007a:76025)

[2] Dagan G., Flow and Transport in Porous Formations, Springer-Verlag, Heidelberg Berlin New York, 465 pp. (1989).

[3] De Zwart B.-R., Investigation of clogging processes in unconsolidated aquifers near water supply wells. Ph.D. thesis, Delft Univ. of Technology. 200 pp. (2007))

[4] De Zwart A.H., Currie P.K., De Boer J., Naeini A.F., Schotting R.J., Experimental and theoretical investigation of clogging processes near production wells using X-ray tomography. SPE paper 116411 (2008).

[5] Drygaś P., Mityushev V., Effective conductivity of unidirectional cylinders with interfacial resistance, Quarterly Journal of Mechanics and Applied Mathematics 62 (3), 235-262 (2009). MR 2524804 
[6] Emets Y.P., Boundary-value problems of electrodynamics of anisotropically conducting media, Naukova dumka, Kiev (1987). (In Russian)

[7] Emets Yu.P., Obnosov Yu.V., An accuratly solvable problem of the mutual effect of inclusions in the theory of heterogeneous media. Journal of Appl. Mech. and Tekhn. Physics, v.31, No 1, 21-29, (1990).(DOI: 10.1007/BF00852740 ) MR.1055573 (92a:78003)

[8] Golubeva O.V., Generalization of the theorem about a circle on seepage flow. Izv. AN USSR, MGhG, No 1, 113 -116 (1966). (In Russian)

[9] Honein E., Honein T., Herrmann G., On two circular inclusions in harmonic problems, Quart. Appl. Math., 50, No 3, 479-499 (1992). MR.1178429 (93f:73031)

[10] Honein E., Honein T., Herrmann G., Energetics of two circular inclusions in anti-plane electrostatics, Int. J. of Solids and Structures., 37, 3667-3679 (2000).

[11] Lee D.K., Image singularity system to represent two circular cylinders of different diameter. Journal of Fluids Engineering, Transactions of the ASME, Volume 122, Issue 4, December 2000, pages 715719 .

[12] Maltseva A.M., Obnosov Yu.V., Rogozin S.V., A generalization of Milne-Thomson theorem on the case of concentric annulus, Uchen. zap. Kazan University, Ser. phys.-math. nauk, 148, book.4, 35-50 (2006). (In Russian)

[13] Maxwell J.C., A Treatise on Electricity and Magnetism, 3rd edn. Oxford University Press, 1, 440 pp. (1904)

[14] Milne-Thomson L.M., Theoretical hydrodynamics. 5th ed., Macmillan (1968).

[15] Milton G.W., The Theory of Composites, Cambridge University Press (2002). MR1899805 (2003d:74077)

[16] Obnosov Yu.V., A generalized Milne-Thomson theorem. Applied Mathematics Letters, 19, 581-586 (2006). MR.2221517 (2007a:30018)

[17] Obnosov Yu.V., Solution of a problem of a seepage fields distribution into infinite porous massif with two circular inclusions. Uch. Zap. Kazan. Gos. Univ., Ser. Fiz.-Mat. Nauki, 148, No. 2, 109-123 (2006). (In Russian)

[18] Obnosov Yu.V., A generalized Milne-Thomson theorem for the case of parabolic inclusion, Applied Mathematical Modelling, 33, 1970-1981 (2009). MR2488259 (2010b:76123)

[19] Obnosov Yu.V., Boundary-value problems of heterogeneous medium theory, Kazan University Press, Kazan (2009). (In Russian)

[20] Obnosov Yu.V., Kasimova R. G., Al-Maktoumi A., Kacimov A.R., Can heterogeneity of the nearwellbore rock cause extrema of the Darcian fluid inflow rate from the formation (the PolubarinovaKochina problem revisited)?, Computers \& Geosciences, Elsevier. Doi:10.1016/j.cageo.2010.01.014 (2010).

[21] Palaniappan D., Electrostatics of two intersecting conducting cylinders. Mathematical and Computer Modelling 36 (7-8), pp. 821-830 (2002). MR1950735 (2003m:78009)

[22] Polubarinova-Kochina P.Ya., Theory of Ground-water Movement, Nauka, Moscow, (1977). (in Russian) MR0670100 (58:32303)

[23] Lord Rayleigh, On the influence of obstacles arranged in rectangular order upon the properties of medium. Phil. Mag. 34, 481-502 (1892).

[24] Strack O.D.L., Groundwater Mechanics. Prentice Hall, Englewood Cliffs (1989).

[25] Wu L., Interaction of two circular cylindrical inhomogeneities under anti-plane shear. Composites Science and Technology 60 (12-13), 2609-2615 (2000) 